\title{
Aggressive silent corticotroph adenoma progressing to pituitary carcinoma. The role of temozolomide therapy
}

\author{
Olga Moshkin, ${ }^{1,9}$ Luis V. Syro, ${ }^{2}$ Bernd W. Scheithauer, ${ }^{3}$ Leon D. Ortiz, ${ }^{4}$ Camilo E. Fadul, ${ }^{5}$ \\ Humberto Uribe, ${ }^{6}$ Ricardo Gonzalez, ${ }^{7}$ Michael Cusimano, ${ }^{8}$ Eva Horvath, ${ }^{9}$ \\ Fabio Rotondo, ${ }^{9}$ Kalman Kovacs ${ }^{9}$
}

${ }^{1}$ Department of Laboratory Medicine, Peterborough Regional Health Centre, Peterborough, ON, Canada, ${ }^{2}$ Department of Neurosurgery, Clinica Medellin and Hospital Pablo Tobón Uribe, Medellin, Colombia, ${ }^{3}$ Department of Laboratory Medicine and Pathology, Mayo Clinic, Rochester, MN, USA, ${ }^{4}$ Section of Neuro-oncology, Instituto de Cancerologia, Clinica Las Americas, Medellin, Colombia; ${ }^{5}$ Section of Hematology/Oncology and Neurology, Dartmouth Hitchcock Medical Center, Lebanon, New Hampshire, USA, ${ }^{6}$ Department of Neurosurgery, Clinica Soma, Medellin, Colombia, ${ }^{7}$ Department of Neurosurgery, Clinica Medellin, Colombia; Departments of ${ }^{8}$ Neurosurgery and ${ }^{9}$ Laboratory Medicine, St. Michael's Hospital, University of Toronto, Toronto, ON, Canada

\begin{abstract}
Temozolomide (TMZ) has recently been recommended as a novel approach in the management of aggressive pituitary tumors. Herein, we present the case of a 43-year-old man with a 20-year history of silent subtype 2 pituitary corticotroph adenoma. Nine surgical resections and radiotherapy had failed to provide a cure. Morphological evaluation of the tumor revealed a mildly pleomorphic adenoma, the cells of which showed low-level cell proliferative activity with Ki67, increased topoisomerase II alpha index and conclusive O-6-methylguanine-DNA methyltransferase (MGMT) as well as vascular endothelial growth factor (VEGF) immunoreactivity. Given its aggressive behavior and failure of conventional therapy, TMZ was administered. The treatment was continued even after MGMT immunopositivity was identified, but failed to decrease MGMT immunoexpression and exerted no morphologic effect. Examination of the lesion after TMZ therapy showed neither morphologic nor immunohistochemical alterations. In our case, TMZ administration, despite changing the TMZ dosing regimen to prompt a drug response, was incapable of depleting MGMT stores.
\end{abstract}

Key words: Carcinoma, MGMT, Pituitary adenoma, Temozolomide, Treatment

Address for correspondence:

Olga Moshkin, M.D., Department of Laboratory Medicine, PRHC, 1 Hospital Drive, Peterborough, On Canada K9J 7C6, Tel.: 1-705-930-6322, Fax: 1-705-876-5008, E-mail: omoshkin@prhc.on.ca

Received 10-08-10, Revised 22-11-10, Accepted 10-01-11

\section{INTRODUCTION}

Temozolomide (TMZ) or Temodal is an antineoplastic alkylating agent now routinely used in the treatment of highly aggressive brain tumors, such as glioblastoma multiforme and nitrosourea, as well as 
procarbazine-resistant anaplastic astrocytoma. ${ }^{1}$ As an imidazotetrazine derivative of the alkylating agent dacarbazine, TMZ undergoes rapid chemical conversion to the active compound, monomethyl triazeno imidazole carboxamide (MTIC). Cytotoxicity of the latter is apparently due to methylation of DNA at the $\mathrm{O}^{6}$ position of guanine, a conversion triggering tumor cell death. ${ }^{2}$ However, some tumors synthesize an enzyme able to repair such DNA damage, thus decreasing the therapeutic efficacy of TMZ. This enzyme is termed O-6-methylguanine-DNA methyltransferase (MGMT) or O-6-alkylguanine-DNA alkyltransferase (AGT or AGAT). ${ }^{1}$ In some tumors, epigenetic silencing of the $M G M T / A G T$ gene prevents synthesis of the enzyme, a process that increases sensitivity to TMZ. ${ }^{3}$ Conversely, tumoral expression of MGMT protein predicts a poor response. $.^{2-4} \mathrm{Re}-$ cently, TMZ has also been found to be effective in the treatment of aggressive atypical pituitary adenomas as well as pituitary carcinomas. ${ }^{5-8}$ Herein, we report the case of a 43-year-old man with a 20-year history of silent subtype 2 pituitary corticotroph adenoma displaying multiple episodes of aggressive tumor regrowth and finally osseous metastases. Treatment modalities included multiple surgeries, radiotherapy (two courses), and TMZ chemotherapy.

\section{CASE REPORT}

A 38-year-old man was referred to the hospital in Medellin, Colombia, in July 2005. Fifteen years prior to his admission, he was found to have a $3-\mathrm{cm}$ pituitary adenoma with suprasellar extension and left cavernous sinus invasion. A partial resection was followed by radiotherapy (5500 cGy). Re-operation for recurrent tumor was undertaken in February 1991. The patient remained asymptomatic for 10 years. In 2001, a second recurrence was diagnosed and prompted craniotomies in 2002 and 2003.

In July 2005, the patient presented with nearly complete blindness in the left eye, left third cranial nerve palsy, and right temporal hemianopsia with a visual acuity of 20/20. Endocrine testing revealed hypopituitarism: luteinizing hormone (LH) $0.51 \mathrm{IU} / 1$ (2-12), free thyroxin (T4) $7.3 \mathrm{pmol} / \mathrm{L}$ (10.2-23.1). Normal or low normal hormone levels included growth hormone $(\mathrm{GH}) 0.06 \mu \mathrm{g} / \mathrm{l}(<5.0)$, insulin-like growth factor 1 (IGF-1) $86.8 \mu \mathrm{g} / \mathrm{L}$ (8-255), prolactin (PRL)
$0.32 \mu \mathrm{g} / \mathrm{l}(0-15)$, follicle stimulating hormone (FSH) $1.61 \mathrm{IU} / \mathrm{l}$ (1-8); thyroid stimulating hormone (TSH) $0.24 \mathrm{mU} / \mathrm{l}(0.5-6.0)$, and cortisol $353 \mathrm{nmol} / \mathrm{l}$ (137689). Magnetic resonance imaging (MRI) revealed a 6-cm sellar mass with clival and left cavernous sinus involvement as well as chiasmal compression (Figure 1, A \& B). The patient underwent transsphenoidal surgery in September 2005, at which time a hard tumor was only partly resected. Three months later, a follow-up MRI scan disclosed residual tumor measuring $60 \%$ of its preoperative size. Given the aggressive behavior of the lesion, accompanied by lack of response to surgical treatment and radiotherapy, TMZ was administered in March 2006. The patient was treated monthly with TMZ, which was given orally for five consecutive days (200 $\mathrm{mg}$ per day) of a 28-day treatment cycle. The patient received 8 cycles of this chemotherapy. During treatment, he

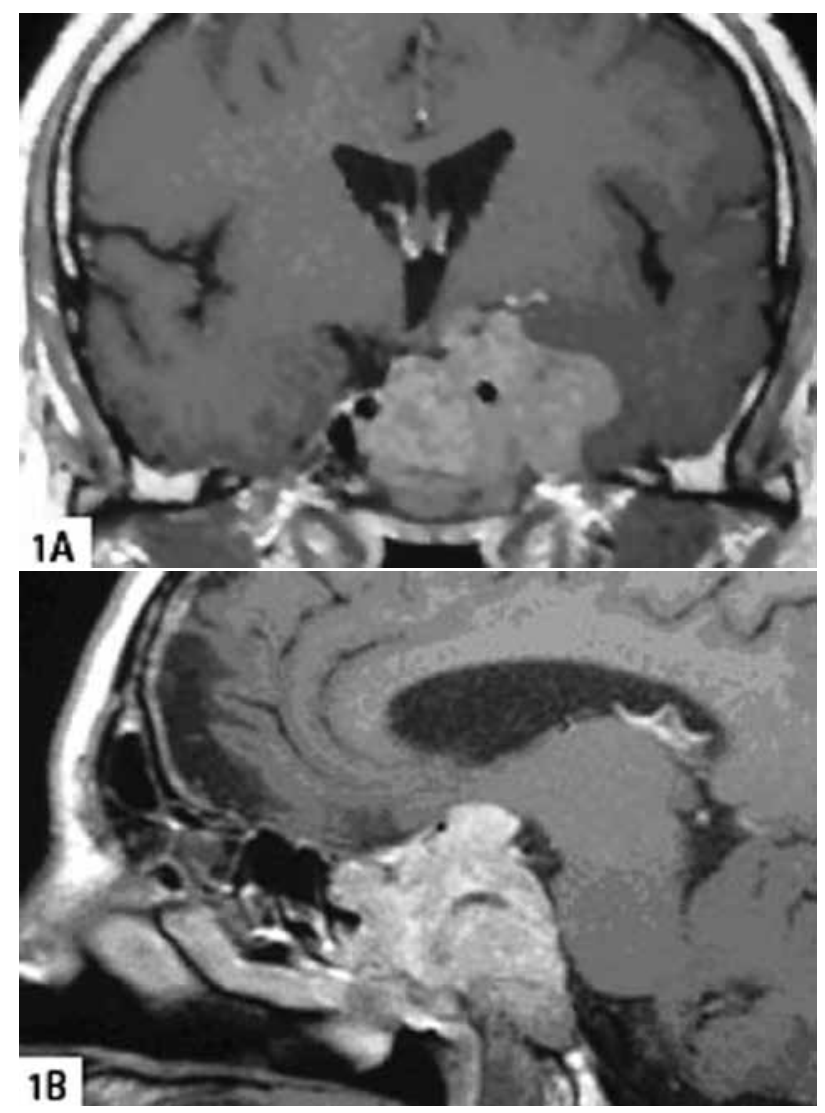

Figure 1. Coronal (A) and sagittal (B) T1-weighted MRI scans with gadolinium enhancement show a sellar and suprasellar tumor with chiasmal compression and left cavernous sinus invasion. 
experienced mild nausea, fatigue, and constipation. No serious hematologic complications were noted, such as neutropenia and thrombocytopenia, which may be seen in less than $10 \%$ of cases. ${ }^{9}$ Follow-up MRI scans again revealed tumor regrowth and chiasmal compression. The patient was twice re-operated on in March and April 2007 by transsphenoidal and frontal approaches, respectively. The last procedure revealed a soft, friable tumor which, on postoperative MRI, was seen to have been near totally removed. The postoperative course was uneventful and TMZ was restarted at the same dose.

Sixteen months later (August 2008), while still on chemotherapy, the patient experienced neck pain. An MRI scan disclosed osseous metastases at spinal levels $\mathrm{C} 2$ and $\mathrm{T} 1$. The former tumor was resected via a transoral approach. Immunohistochemistry revealed widespread nuclear immunoreactivity for MGMT. In an attempt to deplete MGMT, TMZ was restarted at metronomic dose $\left(75 \mathrm{mg} / \mathrm{m}^{2}\right.$ orally, daily, continuously for the next 8 months). Concomitantly, focal radiotherapy (4500 cGy) was administered to the spinal lesions, which resulted in the decrease of lesion size.

Six months later, the patient began complaining of left facial pain and numbness. An MRI scan showed regrowth of the sellar mass with invasion of the left cavernous sinus and clivus. In May 2009, transsphenoidal resection of the sellar mass and its cavernous sinus component succeeded in significantly alleviating facial pain. After the operation, TMZ was discontinued.

Since the tumor failed to respond to TMZ, in May 2009 the patient was started on antiangiogenic agent Bevacizumab (Avastin), a humanized immunoglobulin G1 monoclonal antibody that inhibits vascular endothelial growth factor (VEGF). After 16 months of treatment with Bevacizumab, the patient remains free from tumor progression and metastases and is being followed closely for toxicities and possible relapses.

\section{PATHOLOGY}

Specimens obtained in 2005, 2007, 2008, and 2009 were evaluated by four of the authors (OM, KK, EH, BWS). Specimens were fixed in $10 \%$ buffered formalin, paraffin-embedded, and stained with hema- toxylin and eosin (H\&E) as well as the periodic-acidSchiff (PAS) method. For immunohistochemistry, the streptavidin-biotin-peroxidase complex method was employed using antibodies directed against a spectrum of pituitary hormones (GH, PRL, ACTH, $\beta$-endorphin, FSH, LH, TSH, and $\alpha$-subunit), the endothelial marker CD34 and various biomarkers (VEGF, topoisomerase-II alpha, p53, Ki67, and MGMT, a DNA repair enzyme). Details of immunohistochemical and control methods have been reported in a previous publication. ${ }^{10}$ For electron microscopy, tissues primarily fixed in $2.5 \%$ glutaraldehyde were treated with osmium tetroxide, routinely processed, and embedded in Epon-Araldite.

Light microscopy of all four specimens demonstrated similar morphology. The chromophobic, moderately PAS-positive pituitary adenoma showed a diffuse growth pattern and foci of hemorrhage (Figure 2A). Mild cellular and nuclear pleiomorphism as well as rare mitotic figures were identified. CD34 immunostaining showed the tumor to be markedly vascular, exhibiting irregular and dilated capillaries. No fibrosis or cellular shrinkage was apparent when comparing pre- and post-TMZ-treated specimens.

Immunohistochemistry demonstrated cytoplasmic immunopositivity for ACTH, $\beta$-endorphin as well as $\alpha$-subunit in numerous adenoma cells. Immunostaining was accentuated in perivascular zones. No reactivity for GH, PRL, TSH, FSH, and LH was noted. The Ki67 labeling index decreased somewhat over time, being estimated at 5\% (2005), 2\% (2007 and 2008), and $1 \%$ (2009). The p53 nuclear labeling index varied between 3 to $8 \%$. Over time, the topoisomerase-II alpha index varied from 5\% (2005) to 60\% (2008). Nearly all tumor cell nuclei were immunopositive for MGMT (Figure 2B). There was moderate immunostaining for VEGF in almost all tumor cells (Figure 2C).

Ultrastructurally, the tumor consisted of mediumsize, closely apposed, polyhedral cells (Figure 3). Their largely achromatic, ovoid nuclei possessed moderate to sizable nucleoli. The lucent cytoplasm contained slender parallel stacks of moderately developed rough endoplasmic reticulum and Golgi complexes. Randomly scattered, secretory granules varied in number and focally were numerous. In the majority of cells, they were small (100-150 nm), but scattered 


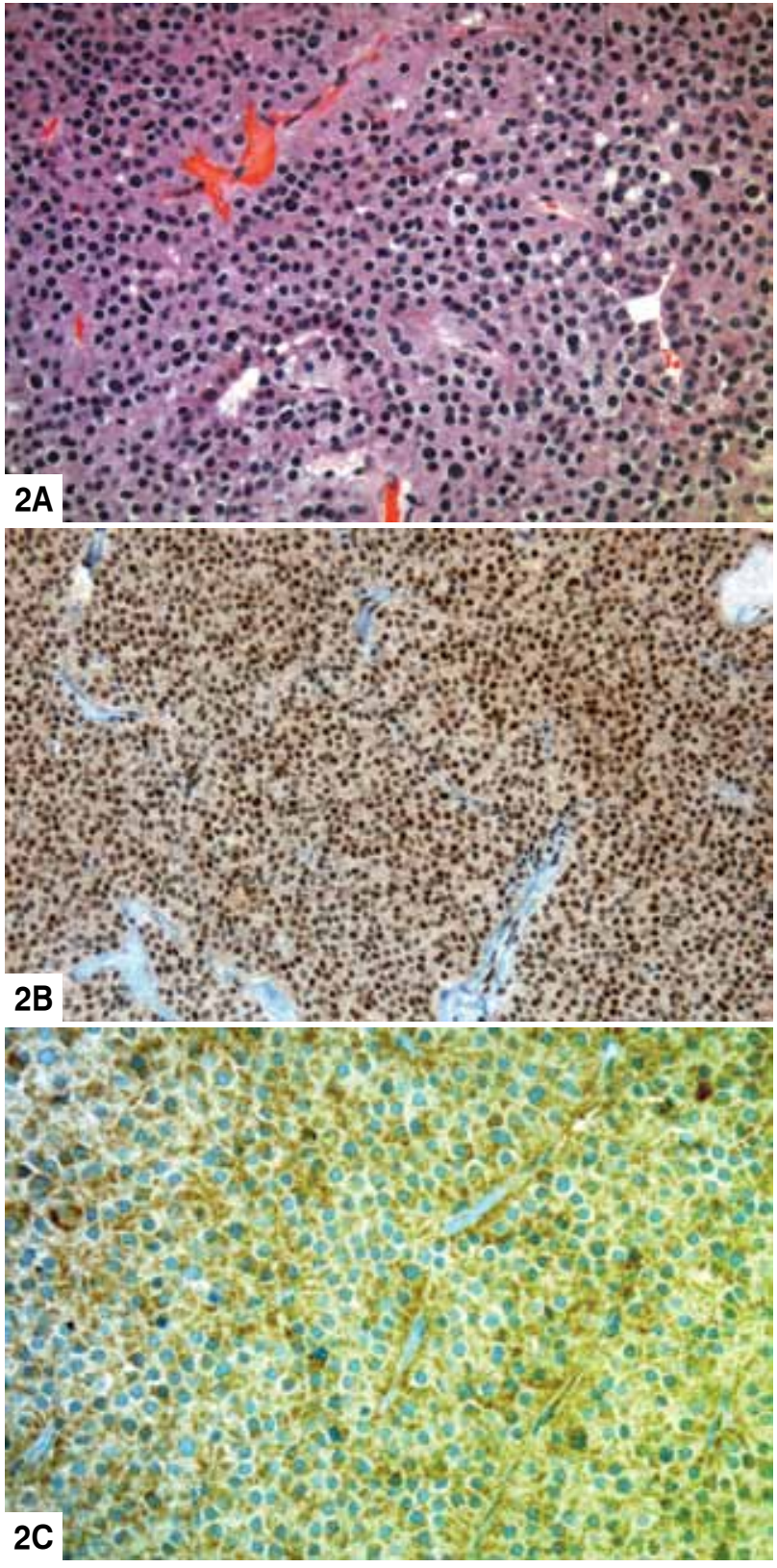

Figure 2. A: Resected adenoma shows sheets of mildly pleiomorphic small cells and rare mitoses $(\mathrm{H} \& \mathrm{E})$; B: Adenoma cells exhibit high nuclear immunopositivity for MGMT. C: Cytoplasmic immunopositivity for VEGF (A-C - original magnification x 200).

cells contained large (up to $300 \mathrm{~nm}$ ) spherical, irregular, and drop-shaped granules. Mitochondria were normal in number and appearance. Many cells contained lysosomes.

Given documented osseous metastases, a diagnosis was made of silent subtype 2 pituitary corticotroph

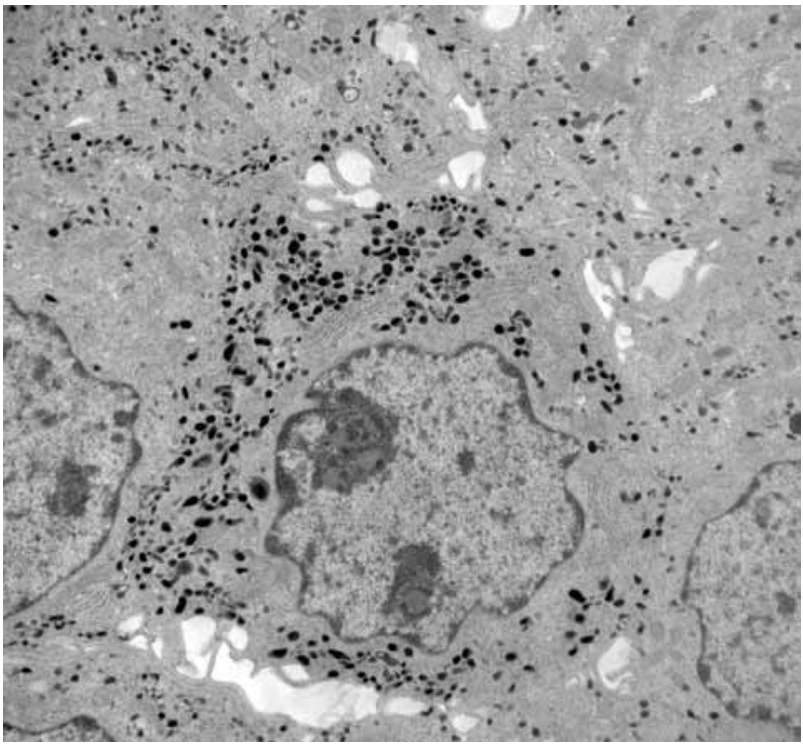

Figure 3. EM: Ultrastructurally, the tumor consists of middle size, polyhedral cells with euchromatic nucleus with moderate to sizable nucleoli. The cytoplasm contains moderately developed rough endoplasmic reticulum and Golgi complexes. Note the numerous small drop-shaped secretory granules characteristic of the tumor-type. (Original magnification $\mathrm{x} 10000$ ).

adenoma transforming to carcinoma.

\section{DISCUSSION}

Treatment of pituitary tumors includes resection and radiation, as well as medical therapy for some endocrine active adenomas. The latter treatment includes dopamine agonists for PRL-producing adenomas and somatostatin analogs for GH-and TSHsecreting tumors. Such standard medical approaches may fail, thus prompting adjuvant chemotherapy. The response of pituitary carcinomas to conventional chemotherapy is often poor and accompanied by a high mortality rate. ${ }^{11}$

Temozolomide is a new antineoplastic agent, an alkylating imidazole tetrazine derivative, the cytotoxic effect of which is based on methylation of DNA at the $\mathrm{O}^{6}$ position of guanine, which serves to inhibit DNA replication and triggers tumor cell death. ${ }^{2}$ The drug is used in the therapy of a variety of tumors including glioblastoma, ${ }^{2,3,12}$ melanoma, ${ }^{9,13}$ and neuroendocrine neoplasms. ${ }^{14}$ In recent years, patients with aggressive pituitary adenomas and carcinomas have also been treated with TMZ. $5,6,8,15-19$ 
The presence within tumor cells of the DNA repair enzyme MGMT, which removes the alkylating groups adducts from the $\mathrm{O}^{6}$ position, induces resistance to $\mathrm{TMZ}^{4,8}$ and indicates a poor therapeutic response to the drug. Patients with such tumors obtain little benefit from TMZ chemotherapy. ${ }^{2}$ Thus, it has been proposed that MGMT expression should be evaluated by immunohistochemical means before administering $\mathrm{TMZ}$ in the setting of aggressive pituitary tumors. ${ }^{15}$

Morphologic changes both before and after TMZ administration, in a case of aggressive PRL-secreting pituitary tumor, have been described. ${ }^{8}$ The main morphologic effects included shrinkage of tumor with associated necrosis, hemorrhage, fibrosis, and neuronal transformation. ${ }^{8}$ In our case, no histologic, immunohistochemical or ultrastructural changes were observed despite multiple courses of TMZ therapy.

It was intriguing that the macroscopic tumor appearance changed over the years from a firm-textured mass (in 2005) to a soft friable tumor after TMZ therapy (in 2007). In spite of macroscopic differences, all four samples resected from 2005 to 2009 displayed similar morphology. The firm consistency of the 2005 tumor is attributed to peritumoral fibrosis, due either to multiple surgeries or to postradiation effects.

There are few reports describing the collective experience with TMZ therapy of aggressive pituitary tumors. Treated tumors have included aggressive PRLsecreting tumors, $, 6,8,15,16$ a functional ACTH adenoma, ${ }^{5}$ a macroprolactinoma progressing to a $\mathrm{GH}$ - and PRLsecreting carcinoma, ${ }^{6}$ non-functional adenomas, ${ }^{5,6}$ and a variety of pituitary carcinomas. ${ }^{6,11,17-19}$

In a preliminary publication, ${ }^{15}$ we reported that the tumor described here, diagnosed as a silent subtype 2 pituitary corticotroph adenoma, was MGMT immunopositive and did not respond to TMZ therapy. The present work expands our study and shows that, despite TMZ administration, the tumor transformed to carcinoma and metastasized to the vertebrae $(\mathrm{C} 2$, T1). Interestingly, topoisomerase-II alpha (topo IIa) was the only biomarker showing a correlation with a clinically aggressive course and malignant transformation. These findings may indicate that the results of different biomarkers should be interpreted with caution. Nonetheless, previous reports ${ }^{20-22}$ have suggested that topo IIa has a prognostic value in several tumor types. Increased topo IIa immunoreactivity is often associated with aggressive clinical behavior and a worse prognosis. Furthermore, it may help to define a role for topoIIa-targeting therapy in patients with aggressive neoplasms.

Recently, Widhalm et $\mathrm{al}^{23}$ published a comprehensive review analyzing alternative treatment for progressive, regrowing, endocrinologically nonfunctioning pituitary adenomas resistant to conventional multimodality therapy. They assessed MGMT expression in 45 such cases, including one group with progressive, regrowing tumors and another group postoperatively tumor-free. The results showed that half of the patients with progressive regrowing lesions exhibited low-level MGMT expression and might thus have been considered candidates for TMZ therapy. In the same paper, it was observed that loss of MGMT expression during tumorigenesis results in impaired DNA repair. Thus, negative or low-level MGMT expression was considered a reliable marker of aggressive tumor behavior. ${ }^{23}$ In our case MGMT was not depleted or down-regulated after malignant transformation.

Silent corticotroph pituitary adenoma is defined as pituitary adenomas showing immunoreactivity for ACTH but without clinical or biochemical evidence of hypercortisolemia. Clinically, the tumors present with mass effects, signs of invasion and, not infrequently, acute tumoral hemorrhage ("apoplexy"). There are aggressive tumors characterized by multiple recurrences and often requiring multimodality treatment. ${ }^{24}$

Herein, we report progressive regrowth and malignant transformation of a silent subtype 2 pituitary corticotroph adenoma in a man with severe visual impairment despite multiple resections and cycles of radiochemotherapy. Morphologic evaluation of the tumor showed only low-level Ki67 labeling, but a high topoisomerase II alpha index and significant VEGF and MGMT immunoreactivity. Given the aggressive course with numerous recurrences and failure of conventional therapies, TMZ treatment was initiated and continued despite high-level MGMT immunopositivity. Examination of the TMZ-treated tumors showed neither morphologic nor immunohistochemical alterations suggestive of a therapeutic response. Thus, our study confirms previous reports that TMZ has no antineoplastic effect in the presence 
of significant MGMT immunopositivity. Apparently, TMZ administration is incapable of depleting MGMT stores resulting in a drug response.

\section{DISCLOSURE STATEMENT}

The authors report no conflicts of interest with regard to the materials or methods used in this study or with respect to our findings.

\section{ACKNOWLEDGMENTS}

The authors thank the Jarislowsky and the CarrLloyd Harris Foundations for their generous support, Mrs. Denise Chase for expert secretarial assistance, Mrs. Judy Marshall for her performance of a literature search, and Dr Rene Ortiz and Sura EPS for their support.

\section{REFERENCES}

1. Newlands ES, Stevens MF, Wedge SR, Wheelhouse RT, Brock C, 1997 Temozolomide: A review of its discovery, chemical properties, pre-clinical development and clinical trials. Cancer Treat Rev 23: 35-61.

2. Yung WK, 2000 Temozolomide in malignant gliomas. Semin Oncol 27: 27-34.

3. Hegi ME, Diserens AC, Gorlia T, et al, 2005 MGMT gene silencing and benefit from temozolomide in glioblastoma. N Engl J Med 352: 997-1003.

4. Levin N, Lavon I, Zelikovitsh B, et al, 2006 Progressive low-grade oligodendrogliomas: Response to temozolomide and correlation between genetic profile and $\mathrm{O}^{6}$-methylguanine DNA methyltransferase protein expression. Cancer 106: 1759-1765.

5. Mohammed S, Kovacs K, Mason W, Smyth H, Cusimano MD, 2009 Use of temozolomide in aggressive pituitary tumors: Case report. Neurosurgery 64: E773-774.

6. Hagen C, Schroeder HD, Hansen S, Hagen C, Andersen M, 2009 Temozolomide treatment of a pituitary carcinoma and two pituitary macroadenomas resistant to conventional therapy. Eur J Endocrinol 161: 631-637.

7. Matsuno A, 2009 Recent trends in the pathophysiology and treatment of pituitary adenomas. Brain Nerve 61: 957-962.

8. Kovacs K, Horvath E, Syro LV, et al, 2007 Temozolomide therapy in a man with an aggressive prolactin-secreting pituitary neoplasm: Morphological findings. Hum Pathol 38: $185-189$.

9. Agarwala SS, Kirkwood JM, 2000 Temozolomide, a novel alkylating agent with activity in the central nervous system, may improve the treatment of advanced metastatic melanoma. Oncologist 5: 144-151.

10. Kovacs K, Stefaneanu L, Horvath E, et al, 1991 Effect of dopamine agonist medication on prolactin producing pituitary adenomas. A morphological study including immunocytochemistry, electron microscopy and in situ hybridization. Virchows Arch A Pathol Anat Histopathol 418: 439-446.

11. Kaltsas GA, Mukherjee JJ, Plowman PN, Monson JP, Grossman AB, Besser GM, 1998 The role of cytotoxic chemotherapy in the management of aggressive and malignant pituitary tumors. J Clin Endocrinol Metab 83: 4233-4238.

12. Lamers LM, Stupp R, van den Bent MJ, et al, 2008 Cost-effectiveness of temozolomide for the treatment of newly diagnosed glioblastoma multiforme: A report from the eortc 26981/22981 nci-c ce3 intergroup study. Cancer 112: 1337-1344.

13. Quirt I, Verma S, Petrella T, Bak K, Charette M, 2007 Temozolomide for the treatment of metastatic melanoma: A systematic review. Oncologist 12: 1114-1123.

14. Ekeblad S, Sundin A, Janson ET, et al, 2007 Temozolomide as monotherapy is effective in treatment of advanced malignant neuroendocrine tumors. Clin Cancer Res 13: 2986-2991.

15. Kovacs K, Scheithauer BW, Lombardero M, et al, 2008 MGMT immunoexpression predicts responsiveness of pituitary tumors to temozolomide therapy. Acta Neuropathol 115: 261-262.

16. Neff LM, Weil M, Cole A, et al, 2007 Temozolomide in the treatment of an invasive prolactinoma resistant to dopamine agonists. Pituitary 10: 81-86.

17. Fadul CE, Kominsky AL, Kingman LS, et al, 2004 Pituitary carcinomas respond to temozolomide. NeuroOnc 6: 374.

18. Lim S, Shahinian H, Maya MM, Yong W, Heaney AP, 2006 Temozolomide: A novel treatment for pituitary carcinoma. Lancet Oncol 7: 518-520

19. Fadul CE, Kominsky AL, Meyer LP, et al, 2006 Longterm response of pituitary carcinoma to temozolomide. Report of two cases. J Neurosurg 105: 621-626.

20. Maruya S, Shirasaki T, Nagaki T, et al, 2009 Differential expression of topoisomerase II alpha protein in salivary gland carcinomas: Histogenetic and prognostic implications. BMC Cancer 9: 72.

21. Nakopoulou L, Zervas A, Lazaris AC, et al, 2001 Predictive value of topoisomerase II alpha immunostaining in urothelial bladder carcinoma. J Clin Pathol 54: 309-313.

22. Lee A, LiVolsi VA, Baloch ZW, 2000 Expression of DNA topoisomerase II alpha in thyroid neoplasia. Mod Pathol 13: 396-400.

23. Widhalm G, Wolfsberger S, Preusser M, et al, 2009 $\mathrm{O}(6)$-methylguanine DNA methyltransferase immunoexpression in nonfunctioning pituitary adenomas: Are progressive tumors potential candidates for temozolomide treatment? Cancer 115: 1070-1080.

24. Karavitaki N, Ansorge O, Wass JA, 2007 Silent corticotroph adenomas. Arq Bras Endocrinol Metabol 51: 1314-1318. 